VOL. $2(1970), 237-245$.

\title{
The Dirichlet boundary value problem for two non-overlapping spheres
}

\section{Dieter K. Ross}

A new method is found for solving the general Dirichlet problem for two non-overlapping spheres of different radius. The expression for the external potential involves hypergeometric functions and is obtained from an infinite set of linear equations. In essence the method makes use of the fact that

$$
(I-A)^{-1}=I+A+A^{2}+A^{3}+\cdots,
$$

where $A$ belongs to a certain class of infinite matrices and $I$ is the unit matrix.

\section{Introduction}

The electrostatic potential extexior to two conducting spheres has been obtained by Kottler [1] and Mitra [2]. These and other authors have made use of Kelvin's method of images which, together with an ingenious set of transformations, leads to the final result. More recently Shail [3] solved the Dirichlet problem for two spheres. He reduced the problem to an integral equation which, after using the transformations proposed by Kottler [1] and Mitra [2], is found to have a simple iterative solution. Now Ross [4], who was concerned with the potential near two dielectric spheres, suggested that a more direct approach could be successful. He expanded the potentials as series of spherical harmonics and showed that the coefficients of these series satisfy an infinite set of linear equations. These can, in principle, be solved by a matrix inversion. In this paper it is found that the general Dirichlet problem for two

Received 22 December 1969. 
non-overlapping spheres leads to an infinite set of linear equations which can be solved explicitly by a matrix inversion. The final expression for the potential is found to be consistent with that given by Shail [3] but the present series is more rapidly convergent.

\section{Expansions for the potential}

Consider two spheres $S_{1}$ and $S_{2}$ which do not overlap, are of radius $a_{1}$ and $a_{2}$ and at separation $R=O_{1} O_{2}$, where $O_{1}$ and $O_{2}$ are the centres of the spheres. [Here the suffix 1 and 2 is used to refer to the first and second sphere respectively.] Let $P$ be a typical point in the vicinity such that. $O_{i} P=r_{i}$ for $i=1$ or 2 and let $\theta_{1}$ and $\theta_{2}$ be the angles $P_{1} \mathrm{O}_{2}$ and $\mathrm{PO}_{2} \mathrm{O}_{1}$. These coordinates are such that $r_{1}=r_{2}$ and $\theta_{1}=\theta_{2}$ for any point $P$ on the median plane. If we suppose that the electrostatic potential $\Phi$ vanishes at infinity and that there are no charges in the region exterior to the two spheres then we may apply Green's theorem to find the potential at the typical point $P$ outside the spheres. Thus

$$
\Phi(P)=\frac{1}{4 \pi} \int\left\{\Phi\left(Q_{i}\right) \frac{\partial}{\partial n}\left(\frac{1}{\rho_{i}}\right)-\frac{1}{\rho_{i}} \frac{\partial \Phi\left(Q_{i}\right)}{\partial n}\right\} d S_{i},
$$

where the integration is over $S_{1}+S_{2}, Q_{i}$ is on the boundary of $S_{i}$ and has spherical polar coordinates $\left(a_{i}, \theta_{i}^{*}, \phi_{i}^{*}\right), \rho_{i}=P Q_{i}, \Phi\left(Q_{i}\right)$ is the potential on the sphere $S_{i}$ and $\partial / \partial n$ denotes differentiation along the inward normal $n$. An expression for $\Phi(P)$ can be found by integrating the above equation with respect to $\phi_{i}^{*}$, remembering that the potential is independent of the azimuthal angle. $\phi$ and using the identities

$$
\rho_{i}^{2}=a_{i}^{2}+r_{i}^{2}-2 a_{i} r_{i} \cos \gamma_{i}
$$

with

$$
\cos \gamma_{i}=\cos \theta_{i} \cos \theta_{i}^{*}+\sin \theta_{i} \sin \theta_{i}^{*} \cos \left(\phi_{i}-\phi_{i}^{*}\right)
$$

together with the addition formula. 
$P_{n}(\cos \gamma)=P_{n}(\cos \theta) P_{n}\left(\cos \theta^{*}\right)+2 \sum_{m=1}^{n} \frac{(n-m) !}{(n+m) !} P_{n}^{m}(\cos \theta) P_{n}^{m}\left(\cos \theta^{*}\right) \cos m\left(\phi-\phi^{*}\right)$

Here $F_{n}^{m}(x)$ is the associated Legendre function as defined in Hobson [5].

In this way we obtain

(1) $\Phi(P)=\sum_{n=0}^{\infty}\left[A_{n}^{(1)}\left(a_{1} / r_{1}\right)^{n+1} P_{n}\left(\cos \theta_{1}\right)+A_{n}^{(2)}\left(a_{2} / r_{2}\right)^{n+1} P_{n}\left(\cos \theta_{2}\right)\right]$

where

$$
A_{n}^{(i)}=\frac{1}{2} \int_{0}^{\pi} X_{n}\left(Q_{i}\right) P_{n}\left(\cos \theta_{i}^{*}\right) \sin \theta_{i}^{*} d \theta_{i}^{*}
$$

and

$$
X_{n}\left(Q_{i}\right)=a_{i} \partial \Phi\left(Q_{i}\right) / \partial n-n \Phi\left(Q_{i}\right)
$$

By Schwarz's inequality it follows that

$$
\begin{aligned}
\left(A_{n}^{(i)}\right)^{2} \leq \frac{1}{4} \int_{0}^{\pi} X_{n}^{2}\left(Q_{i}\right) \sin \theta_{i}^{*} d \theta_{i}^{*} \cdot \int_{0}^{\pi} P_{n}^{2}\left(\cos \theta_{i}^{*}\right) \sin \theta_{i}^{*} d \theta_{i}^{*} & \\
& =[1 /(4 n+2)] \int_{0}^{\pi} X_{n}^{2}\left(Q_{i}\right) \sin \theta_{i}^{*} d \theta_{i}^{*} .
\end{aligned}
$$

Since the potential and its gradient normal to the boundary of $S_{i}$ are integrable we obtain the result $\left|n^{-\frac{1}{2} A}{ }_{n}^{(i)}\right|$ is bounded for all $n$. Thus the two series in (1) representing $\Phi(P)$ are absolutely and uniformly convergent in the region exterior to the two spheres, except perhaps at the points where $\theta_{i}=0$ or $\pi$.

\section{The boundary value problem}

We shall consider the Dirichlet boundary value problem where the potential outside the two spheres satisfies the conditions:

$$
\Phi=B_{i} P_{k_{i}}\left(\cos \theta_{i}\right) \text { on } r_{i}=a_{i} \text { for } i=1,2 \text {, }
$$

and 


$$
\Phi \rightarrow 0 \text { as } r_{1} \text { and } r_{2} \rightarrow \infty
$$

In order to apply these conditions in equation (1) we shall need the shift formula of zonal harmonics, namely

$$
\frac{P_{n}\left(\cos \theta_{2}\right)}{r_{2}^{n+1}}=\frac{1}{R^{n+1}} \sum_{m=0}^{\infty}\left(\begin{array}{c}
m+n \\
n
\end{array}\right)\left(\frac{r_{1}}{R}\right)^{m} P_{m}\left(\cos \theta_{1}\right)
$$

which is valid for $\left|r_{1}\right|<R$, and a similar expression with the suffices $I$ and 2 interchanged. These equations are given in Hobson [5] although he defined the angles $\theta_{i}$ in a slightly different way.

From the boundary conditions $(2 a)$ we get, after substitution of the above result into equation (1), rearranging the absolutely convergent series and making use of the orthogonality of the Legendre polynomials

$$
B_{1} \delta_{m, k_{1}}=A_{m}^{(1)}+t_{2} \sum_{s=0}^{\infty} p_{m, s} A_{s}^{(2)}
$$

and

(4) $\quad B_{2} \delta_{m, k_{2}}=A_{m}^{(2)}+t_{1} \sum_{s=0}^{\infty} p_{s, m_{s}}^{(1)}$ for $m=0,1,2, \ldots$,

where

$$
p_{m, n}=\left(\begin{array}{c}
m+n \\
n
\end{array}\right) t_{1}^{m} t_{2}^{n}, \quad t_{i}=a_{i} / R \text { and } 0 \leq t_{1}+t_{2}<1 .
$$

Here $\delta_{i, j}$ is the Kronecker delta function. Elimination of $A_{m}^{(1)}$ from equations (3) and (4) leads to

$$
B_{2} \delta_{n, k_{2}}-B_{1} t_{1} p_{k_{1}, n}=\sum_{s=0}^{\infty}\left\{\delta_{n, s}-q_{n, s}^{(2)}\right\}_{s}^{(2)}
$$

where

$$
q_{n, s}^{(2)}=t_{1} t_{2} \sum_{m=0}^{\infty} p_{m, n^{p}, s}
$$

Similarly, on elimination of $A_{m}^{(2)}$ we obtain 


$$
B_{1} \delta_{n, k_{1}}-B_{2} t_{2} p_{n, k_{2}}=\sum_{s=0}^{\infty}\left\{\delta_{n, s}-q_{n, s}^{(1)}\right\}_{s}^{(1)}
$$

where

$$
q_{n, s}^{(1)}=t_{1} t_{2} \sum_{m=0}^{\infty} p_{n, m^{p} s, m} \text {. }
$$

\section{Inverse of the infinite matrices}

Now it is a simple matter to verify that the sums of the rows and columns of the matrices $q_{n, s}^{(i)}$ are each of modulus less than 1 . Hence, from Theorem 2.4,I of Cooke [6], it follows that $\delta_{n, s}-q_{n, s}^{(i)}$ has the unique and two-sided reciprocal

$$
I+\sum_{m=1}^{\infty} Q_{i}^{m}, Q_{i}=\left(q_{n, s}^{(i)}\right), I \text { the unit matrix . }
$$

The next step is to find a sufficiently simple expression for the various powers of the matrices $Q_{i}$. We begin with three lemmas.

LEMMA 1. Let $\left\{U_{n}\right\}$ with $n=1,2,3, \ldots$ be a set of functions of $\left(t_{1}, t_{2}\right)$ which satisfy the difference equations

$$
U_{2 n+1}=U_{2 n}-t_{1}^{2} U_{2 n-1}
$$

and

$$
U_{2 n}=U_{2 n-1}-t_{2}^{2} U_{2 n-2}
$$

where $t_{1}$ and $t_{2}$ are positive constants such that $0 \leq t_{1}+t_{2}<1$ and the initial conditions are

$$
U_{1}=1, \quad U_{2}=1-t_{2}^{2} .
$$

Then the following deductions can be made:

(a) if $t_{1}=t_{2}=0$ then $U_{n}=1$ for all $n$,

(b) if $t_{1}$ and $t_{2}$ are not zero then

$$
U_{2 n-1}=\left(t_{1} t_{2}\right)^{n-1} \sinh n \mu / \sinh \mu
$$


and

$$
U_{2 n}=t_{1}^{n} t_{2}^{n-1}\left\{t_{1} \sinh n \mu+t_{2} \sinh (n+1) \mu\right\} / \sinh \mu
$$

with $\mu$ positive and given by

$$
\cosh \mu=\left(1-t_{1}^{2}-t_{2}^{2}\right) /\left(2 t_{1} t_{2}\right) .
$$

(c) $U_{2 n+1}=\left(1-t_{2}^{2}\right) U_{2 n-1}-t_{1}^{2}\left\{U_{2 n-1}^{2}-t^{2 n-2} t_{2}^{2 n}\right\} / U_{2 n}$,

(d) $0 \leq t^{2} U_{2 n-1}<\left(1-t_{2}^{2}\right) U_{2 n}$,

where the equality is only possible when $t_{1}=0$.

The proof of $(b)$ is based on the general theory of linear difference equations as discussed in the book by Durell and Robson [7] and the conclusion in (c) may be obtained by a direct substitution of (b). The inequalities in (d) follow from the fact that

$0 \leq t_{2}^{2} U_{2 n} / U_{2 n+1}=t_{2}\left\{t_{1} \sinh n \mu+t_{2} \sinh (n+1) \mu\right\} / \sinh (n+1) \mu<t_{2}\left(t_{1}+t_{2}\right)<1$. Combining this with equation (10) gives the required result.

LEMMA . 2.

$$
\sum_{k=0}^{\infty}\left(\begin{array}{c}
i+k \\
k
\end{array}\right) x^{k}{ }_{2} F_{1}(-k,-j ; 1 ; z)=\frac{(1-x+x z)^{j}}{(1-x)^{i+j+1}}{ }_{2} F_{1}\left(-i,-j ; 1 ; \frac{x z}{1-x+x z}\right) \text {, }
$$

where ${ }_{2} E_{1}(a, b ; c ; z)$ is the hypergeometric function, $i$ and $j$ are positive integers and $|x|$ and $|z|$ are each less than 1 . This identity can be proved by using the power series expansion

$$
{ }_{2} F_{1}(-k,-j ; 1 ; z)=\sum_{n=0}^{\min (k, j)} \frac{(-k)_{n}(-j) z^{z^{n}}}{(-1)_{n} n !},
$$

given in Bateman [8], and changing the order of the surmation on the left-hand side of the identity.

\section{LEMMA 3.}

$$
\begin{aligned}
\sum_{k=0}^{\infty} x^{k}{ }_{2} F_{1}(-i,-k ; 1 ; A) & { }_{2} F_{1}(-k,-j ; 1 ; B) \\
& =(1-x+A x)^{i}(1-x+B x)^{j}{ }_{2} F_{1}(-i,-j ; 1 ; S) /(1-x)^{i+j+1}
\end{aligned}
$$


where

$$
S=x A B /[(1-x+A x)(1-x+B x)],
$$

$i$ and $j$ are positive integers and $|x|,|A|$ and $|B|$ are each less than 1 .

This identity can be proved by using the expansion

$$
{ }_{2} F_{1}(-k,-j ; 1 ; B)=(1-B)^{j+k+1} \sum_{m=0}^{\infty}\left(\begin{array}{c}
k+m \\
m
\end{array}\right)\left(\begin{array}{c}
j+m \\
m
\end{array}\right) B^{m},
$$

changing the order of the summation and applying Lemma 2 appropriately.

We shall now prove, by induction, that the matrix of coefficients of the $n$-th power of $q_{n, s}^{(1)}$ is given by

$$
t_{1}^{m+s}\left(t_{1} t_{2}\right)^{n} U_{2 n-1}^{m+s}{ }_{2} F_{1}\left(-m,-s ; 1 ; t_{1}^{2 n-2} t_{2}^{2 n} / U_{2 n-1}^{2}\right) / U_{2 n}^{m+s+1}
$$

for $n=1,2,3, \ldots$.

This result is trivial for $n=1$ since

$$
\begin{aligned}
q_{m, s}^{(1)} & =t_{1}^{m+s}\left(t_{1} t_{2}\right) \sum_{n=0}^{\infty}\left(\begin{array}{c}
m+n \\
n
\end{array}\right)\left(\begin{array}{c}
n+s \\
s
\end{array}\right) t_{2}^{2 n} \\
& =t_{1}^{m+s}\left(t_{1} t_{2}\right){ }_{2} E_{1}\left(-m,-s ; 1 ; t_{2}^{2}\right) /\left(1-t_{2}^{2}\right)^{m+s+1} .
\end{aligned}
$$

This follows from a transformation formula for the hypergeometric function given in Bateman [8].

Let us consider the matrix product

$$
\begin{gathered}
\sum_{k=0}^{\infty}\left\{q_{m, k}^{(1)}\right\}^{n} q_{k, s}^{(1)}=\frac{t_{1}^{m+s}\left(t_{1} t_{2}\right)^{n+1} U_{2 n-1}^{m}}{U_{2 n}^{m+1} U_{2}^{s+1}} \sum_{k=0}^{\infty}\left\{\frac{t_{1} U_{2 n-1}}{U_{2 n} U_{2}}\right\}^{k} \\
{ }_{2} F_{1}\left(-m,-k ; t_{1}^{2 n-2} t_{2}^{2 n} / U_{2 n-1}^{2}\right){ }_{2} F_{1}\left(-k,-s ; 1 ; t_{2}^{2}\right) ;
\end{gathered}
$$

then from Lemmas 1 and 3 it follows, after a considerable amount of algebra, that

$$
\sum_{k=0}^{\infty}\left\{q_{m, k}^{(1)}\right\}^{n} q_{k, s}^{(1)}=\left\{q_{m, s}^{(1)}\right\}^{n+1}
$$

This completes the proof. 
Equation (15) may now be used to find the inverse of the matrix $\delta_{n, s}-q_{n, s}^{(1)}$ which, when applied to equation (8), determines the set of constants $A_{S}^{(1)}$. In particular, if we make use of the symbol

$$
U_{m}^{*}=U_{m}\left(t_{2}, t_{1}\right) \text { when } U_{m}=U_{m}\left(t_{1}, t_{2}\right)
$$

then

$$
\left\{\begin{array}{rl}
A_{s}^{(1)}= & B_{1} \delta_{s, k_{1}}-B_{2}\left(\begin{array}{c}
s+k_{2} \\
s
\end{array}\right) t_{1}^{s} t_{2}^{1+k_{2}}+B_{1} t^{s+k_{1}} \sum_{n=1}^{\infty}\left(t_{1} t_{2}\right)^{n} U_{2 n-1}^{s+k_{1}} \\
& { } F_{1}\left(-s,-k_{1} ; 1 ; t_{1}^{2 n-2} t_{2}^{2 n} / U_{2 n-1}^{2}\right) / U_{2 n}^{1+s+k_{1}}-B_{2} t_{1}^{s} t_{2}^{1+k_{2}} \\
& \sum_{n=1}^{\infty}\left(t_{1} t_{2}\right)^{n} U_{2 n}^{s} U_{2 n}^{k_{2}}{ }_{2} F_{1}\left(-s,-k_{2} ; 1 ; t_{1}^{2 n} t_{2}^{2 n} / U_{2 n} U_{2 n}^{*}\right) / U_{2 n+1}^{1+s+k_{2}}
\end{array} .\right.
$$

The formula for $A_{B}^{(2)}$ can be deduced from the above by interchanging the suffices 1 and 2 . The equations for $A_{s}^{(i)}$ can be simplified by making use of the transformations

$$
\sinh \lambda_{1}=t_{1} \sinh \mu \text { and } \sinh \lambda_{2}=t_{2} \sinh \mu .
$$

If we combine these with equation (14) then we find ihat

$$
\cosh \lambda_{1}=t_{2}+t_{1} \cosh \mu, \cosh \lambda_{2}=t_{1}+t_{2} \cosh \mu \text { and } \mu=\lambda_{1}+\lambda_{2},
$$

and on substitution of these identities into (13) we obtain

$$
U_{2 n}=t_{1}^{n} t_{2}^{n-1} \sinh \left(n \mu+\lambda_{2}\right) / \sinh \mu \text {. }
$$

Finally equation (17) becomes

$$
\begin{aligned}
& A_{s}^{(1)}=\sinh \lambda_{2}\left(B_{1} \sum_{n=1}^{\infty} \frac{\sinh ^{s+k_{1}} n \mu}{\sinh ^{1+s+k_{1}}\left(n \mu+\lambda_{2}\right)}{ }_{2} F_{1}\left(-s,-k_{1} ; 1 ; \frac{\sinh ^{2} \lambda_{2}}{\sinh ^{2} n \mu}\right)\right. \\
& \left.-B_{2} \sum_{n=1}^{\infty} \frac{\sinh ^{8}\left(n \mu+\lambda_{1}\right) \sinh ^{k_{2}}\left(n \mu+\lambda_{2}\right)}{\sinh ^{1+s+k_{2}}(n+1) \mu} F_{1}\left(-s,-k_{2} ; 1 ; \frac{\sinh \lambda_{1} \sinh \lambda_{2}}{\sinh \left(n \mu+\lambda_{1}\right) \sinh \left(n \mu+\lambda_{2}\right)}\right)\right) . \\
& \text { It is a simple matter to verify that this result is consistent with } \\
& \text { whence } k_{1}=k_{2}=0 \text { and the hypergeometric function disappears }
\end{aligned}
$$


altogether. This work is also in agreement with the result obtained by

Shail [3] who did not express the coefficients $A_{s}^{(i)}$ in terms of hypergeometric functions and did not make use of their properties in order to improve the convergence rate.

\section{References}

[1] F. Kottler, "Elektrostatik der Leiter", Handbuch der Physik XII, 349-492, especially 428-453 (Julius Springer, Berlin, 1927).

[2] Samarendra Kumar Mitra, "A new method of solution of the boundary value problems of Laplace's equation relating to two spheres", Bul2. Calcutta Math. Soc. 36 (1944), 31-39.

[3] R. Shail, "On some axisymmetrical two sphere potential problems", Mathematika 9 (1962), 57-70.

[4] D.K. Ross, "The potential due to two point charges each at the centre of a spherical cavity and embedded in a dielectric medium", Austral. J. Phys. 21 (1968), 817-822.

[5] E.W. Hobson, The theory of spherical and ellipsoidal harmonics (Cambridge University Press, Cambridge, 1931).

[6] Richard G. Cooke, Infinite matrices and sequence spaces (Macmillan, London, 1950).

[7] C.V. Durrell and A. Robson, Advanced algebra, II (G. Bell, London, 1937).

[8] Arthur Erdélyi, Wilhelm Magnus, Fritz Oberhettinger and Francesco G. Tricomi, Higher transcendental functions, $I$. Based, in part, on notes left by Harry Bateman. (McGraw-Hill, New York, Toronto, London, 1953).

La Trobe University,

Bundoora, victoria. 